\title{
Susceptibilités génétiques, apports lipidiques et prise de poids
}

\section{Karine CLEMENT}

Service de nutrition, Hôpital de la PitiéSalpêtrière, Inserm UMRS 872, Équipe 7 Centre de recherche des Cordeliers, Université Paris 6 $<$ karine.clement@psl.aphp.fr>

\begin{abstract}
Human obesity is a multifactorial disease (except few monogenic cases) determined by the interaction of predisposing factors, genetic and environmental ones. The purpose of this review is to present some examples of genetic studies in obesity and some of the difficulties bound to these researches: the multiplicity of environmental and genetic factors to be dealt with, the power of population studies, the accuracy of collected phenotypes and the limits of our tools for dealing with this complexity.
\end{abstract}

Key words: interactions of genes and environment
La notion d'une susceptibilité individuelle à l'obésité est ancienne. En 1852, dans un traité de médecine générale, Hufeland faisait remarquer : «En général, une disposition congénitale a une grande influence; ainsi certaines personnes restent maigres malgré la nourriture la plus riche, et d'autres deviennent obèses alors qu'elles sont soumises à restriction. » Cette remarque est un peu schématique, mais Hufeland soulignait déjà l'inégalité entre les sujets face à la prise de poids. En 1923, Davenport notait que «l'obésité a tendance à se concentrer au sein de certaines familles", illustrant le rôle essentiel de l'environnement familial et des facteurs biologiques, éventuellement génétiques. Naturellement, une telle hypothèse, celle de la susceptibilité individuelle à la prise de poids et à I'obésité aux apports alimentaires, et en particulier aux apports lipidiques, nécessitait d'être mise à l'épreuve. Les études épidémiologiques menées ont apporté des arguments.

\section{Les études d'épidémiologie génétique}

Ce sont les analyses de populations, et singulièrement les études d'épidémiologie génétique, qui ont constitué des premières contributions de la mise à l'épreuve de cette hypothèse. L'analyse des jumeaux élevés ensemble ou séparément grâce aux registres d'Europe du Nord, I'analyse des populations migrantes, comme des populations des atolls du Pacifique (Tokeluens), les populations migrantes aux États-Unis (d'origine asiatique ou mexicaine par exemple) ont été contributives et la contribution relative des facteurs génétiques et d'environnement calculée. Ainsi I'« héritabilité génétique ", permet de calculer la part de variation d'un trait, lié à l'obésité - masse grasse, corpulence - attribuable à des facteurs génétiques.

Mais comment en dégager avec confiance les parts respectives de l'environnement et des facteurs génétiques notamment dans les études familiales? De fait, les chiffres présentés sur I'héritabilité génétique par exemple furent I'objet de nombreux débats chez les spécialistes de l'épidémiologie génétique.

La célèbre étude de suralimentation menée par Claude Bouchard au début des années 1990 au Québec a été déterminante. II s'agissait de convier des jumeaux monozygotes à consommer 1000 calories de plus par jour que leur consommation habituelle pendant environ 100 jours. À l'issue de l'étude, une inégalité de la prise de poids et de masse grasse fut bien constatée entre les sujets mais la variabilité des traits modifiés était plus faible au sein d'une même paire de jumeaux (partageant le même patrimoine génétique) qu'entre les paires. Un résultat qui effectivement suggérait bien l'existence d'une susceptibilité individuelle, génétique, à la suralimentation. Des résultats semblables ont été obtenus lors de la perte de poids chez des jumeaux soumis à une sousalimentation.
Autre étude aux résultats passionnants, celle conduite par Eric Ravussin auprès des Indiens Pima et en Arizona. Au Mexique, ces Indiens vivent dans les montagnes de la Sierra Madre, près de Mexico. Ils sont essentiellement cultivateurs, et leur mode de vie traditionnel comportant un apport énergétique moins important et une moindre consommation alimentaire (moindres apports en lipides) que ceux vivants en Arizona (figure 1). Ils présentent néanmoins un taux d'obésité compris entre $13 \%$ à $17 \%$, soit un chiffre significatif de leur prédisposition (quand on les rapproche par exemple de la fréquence de l'obésité en Europe). En Arizona (Etats-Unis), où ces Indiens sont sédentarisés et où leur consommation lipidique représente plus de $35 \%$ de l'énergie totale consommée, entre $70 \%$ et $80 \%$ des sujets sont obèses, soient des taux très élevés, même pour la population américaine. II faut noter également des différences importantes dans les niveaux d'activité physique entre des Indiens Pima du Mexique et ceux d'Arizona.

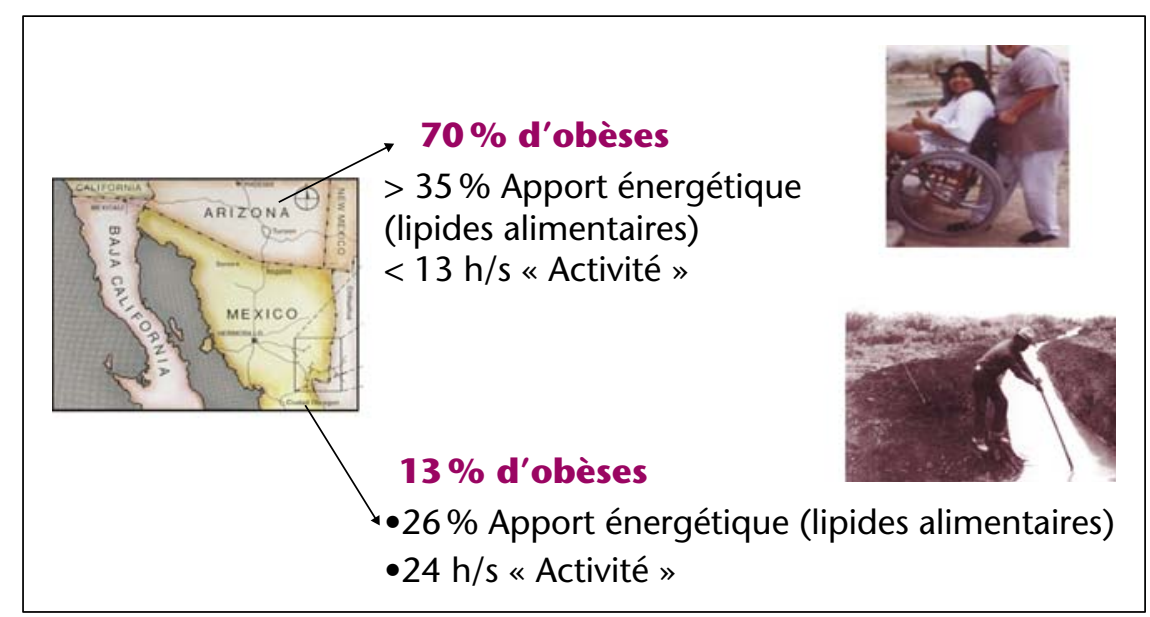

Figure 1. Susceptibilités génétiques: exemple de population prédisposée, les Indiens Pima. D’après Ravussin E, Valencia ME, Esparza I et al. Effects of a traditional lifestyle on obesity in Pima Indians. Diabetes Care 1994. 


\section{Les outils moléculaires}

Avec la possibilité de modifier l'expression des gènes (transgenèse), la voie a été ouverte à l'analyse de gènes de susceptibilité aux lipides alimentaires. Aujourd'hui, très régulièrement sont publiés des models d'invalidation ou de surexpression génique rendant des souris très sensibles ou au contraire très résistantes à la prise de poids. Parallèlement, l'analyse du rôle des polymorphismes génétiques au travers des larges études de population s'est développée.

\section{Les modèles animaux}

Nous ne reviendrons pas sur les données présentées par Jean-Michel Beylot concernant les voies qui conduisent à l'accumulation de triglycérides (figure 2) dans l'adipocyte puis à leur libération sous forme d'acides gras et de glycérol. De nombreux acteurs de ces voies ont été mis en avant dans des modèles animaux impliquant des gènes sélectifs du transport, stockage et de l'hydrolyse des acides gras. Ont été également réalisées chez le rongeur des invalidations, des transgénèses de gènes sélectifs codant pour des facteurs de transcription, comme par exemple les PPAR (récepteur au facteur activé de prolifération des peroxysomes), dont on connaît le rôle important dans la lipogenèse.

Naturellement, à l'échelle de l'animal entier, on ne peut invalider la totalité des gènes codant pour plusieurs facteurs de transcription essentiels et ainsi bloquer la synthèse d'acides gras, les animaux n'y survivent généralement pas. Grâce aux modèles conditionnels, invalidant spécifiquement tel ou tel gène au sein de tissus donnés, comme le tissu adipeux, on peut en étudier les effets spécifiques.

Le tableau 1 présente des résultats obtenus dans plusieurs modèles. On reconnaît la lipoprotéine lipase ( $L P L)$, les récepteurs $C D 36$, la périlipine, ALB/AP2, lipase hormono-sensible (HSL). Leur rôle est précisé ainsi que leurs effets sur le plan de la modification de la masse adipeuse du rongeur. On s'aperçoit que pour la lipoprotéine lipase (LPL), par exemple, l'effet est obtenu en présence d'un «fond » génétique spécifique, celui des souris ob/ob leptinodéficientes et génétiquement obèses. Pour la lipase hormono-sensible (HSL), hormone clé pour de la lipolyse, l'absence d'effet constaté a conduit à soupçonner d'autres facteurs intervenant dans l'hydrolyse des triglycérides.

Les récepteurs nucléaires sont un autre grand volet de ces études. Dans les modèles hétérozygotes par croisement soumis au régime gras, ces animaux se révèlent souvent protégés de I'obésité. L'invalidation de ces gènes, et les effets constatés dans les modèles animaux, fournit ainsi tout un éventail de gènes candidats pour les études génétiques chez l’homme.

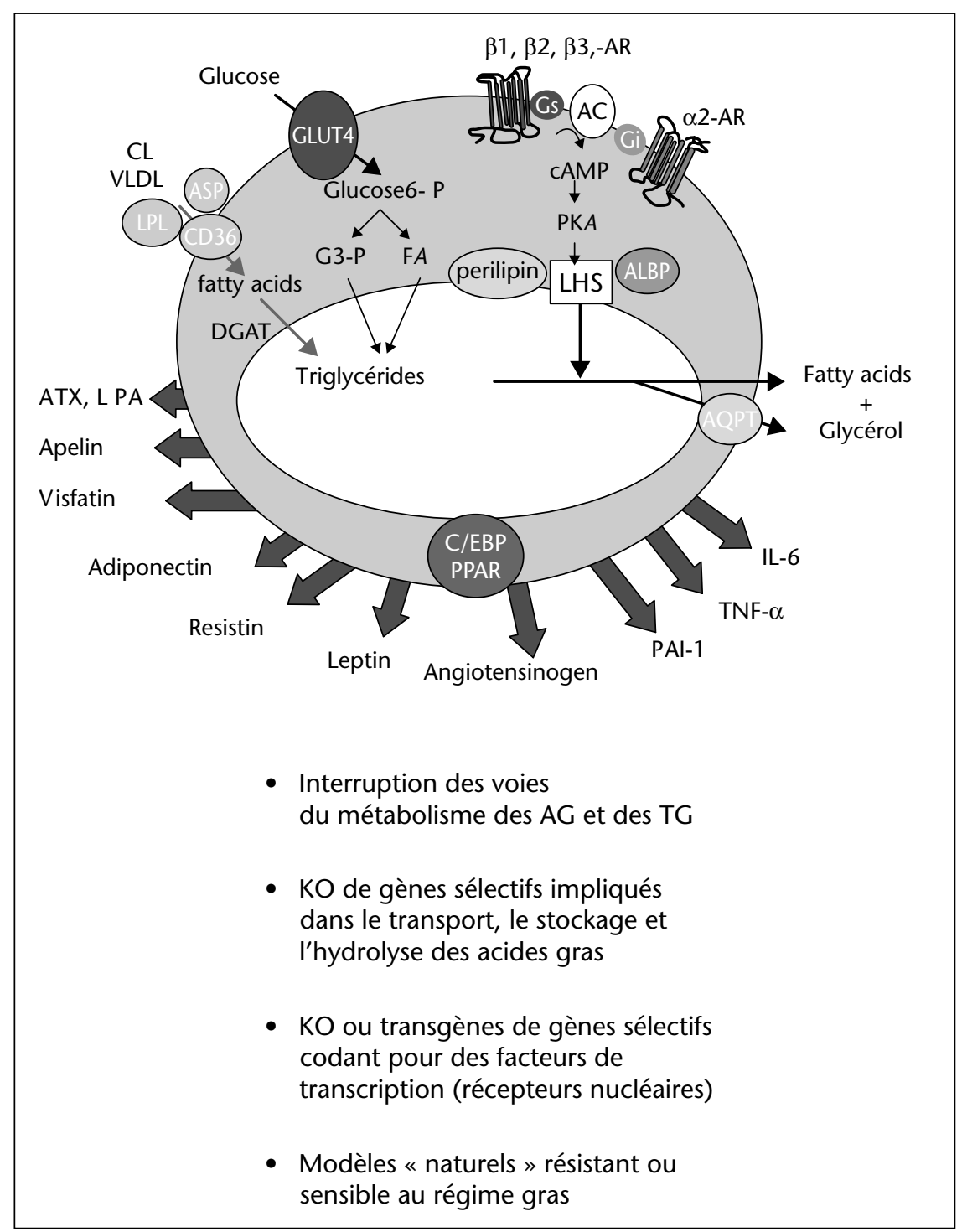

Figure 2. Interactions gènes-lipides alimentaires. Tissu adipeux des modèles animaux. D'après P. Valet. Obesity Genomics and post genomics. Ed T K Clément \& Thorkild Sorensen, Healthcare 2007.

Tableau 1. Modèles murins d'invalidation (gènes du métabolisme des acides gras).

\begin{tabular}{|c|c|c|c|}
\hline Gène & Rôle & Masse grasse & Conséquence \\
\hline $\begin{array}{l}\text { LPL -/- } \\
\text { Cell. adipeuse }\end{array}$ & Hydrolyse TG & Seulement si sur « fond » ob/ob & $\begin{array}{l}\text { Prise poids, } \\
\text { contenu } \\
\text { en lipide }\end{array}$ \\
\hline CD36 & Transport AG & - & Résistant au « DIO » \\
\hline ASP & Synthèses TC & $25 \%$ & $\begin{array}{l}\text { Dépôt graisse régime HF et } \\
\text { LF (modeste) }\end{array}$ \\
\hline DGAT & Synthèses TC & $50 \%$ & $\begin{array}{c}\text { Résistant au « DIO », RAS } \\
\text { Chow } \\
\text { Elévation DER }\end{array}$ \\
\hline Périlipine & Lipid droplet coating & $50-70 \%$ & Résistant au DIO \\
\hline ALBP/AP2 & Interaction avec HSL & - & Protection contre IR (HF) \\
\hline HSL & Hydrolyse TC & - & - \\
\hline
\end{tabular}


Il existe également des modèles d'obésité polygénique. Par exemple, les souris AKR/J se révèlent extrêmement sensibles aux régimes gras alors que soumises à un régime hypergras les souris SWR révèlent une forte résistance.

Ces résultats fournissent au généticien de la souris des modèles, lui permettant $d^{\prime}$ analyser les régions du génome associées à une susceptibilité particulière, régions appelées les régions QTL (quantitative trait loci), régions liées à des phénotypes d'obésité ou du métabolisme lipidique. Ces travaux nombreux issus des analyses génétiques chez les rongeurs n'ont pas encore permis de dégager l'influence majeure de certains gènes candidats situés dans ces régions QTL.

\section{Les études génétiques chez l'homme: contexte}

La situation se complique chez I'humain car le plus souvent nous ne sommes pas en présence d'affections monogéniques, avec mutation d'un seul gène entraînant le développement rapide de l'obésité. Le phénotype des sujets présentant ces anomalies génétiques rares, en particulier sur la voie leptine et la voie des mélanocortines, est illustratif. Ces mutations sont associées dès les premières semaines de vie à une obésité extrêmement sévère. Ces cas mis à part dans les situations les plus fréquentes, l'obésité nous confronte à une situation caractérisée par des interactions entre de nombreux gènes candidats et les modifications environnementales.

Avant d'évoquer les gènes candidats il est important de situer le contexte de ces études génétiques : que peut-on en dire raisonnablement? Quelles difficultés, en particulier méthodologiques, obligent-elles à surmonter ou à contourner?
Sans revenir sur le développement «naturel » de l'obésité, il convient de comprendre que les interactions gènes/environnement interviennent vraisemblablement à différents niveaux. Pour chaque phase du développement (prise de poids, stabilisation, chronicisation) (figure 3) les interactions gènes/gènes ou gènes/ environnement peuvent être extrêmement différentes et donc délicates à étudier. Finalement le phénotype de résistance à la perte de poids est bien souvent ce qui caractérise l'obésité installée. II est souvent difficile de juger de l'histoire pondérale très hétérogène des personnes impliquées dans les études d'épidémiologie génétique, et de caractériser avec précision l'ensemble des facteurs d'environnement. L'autre aspect associé au développement de I'obésité porte sur le caractère épigénétique de cette pathologie, également bien difficile à étudier chez l'homme.

L'émergence des outils informatiques, la mise à disposition des informations issues du séquençage du génome ont par ailleurs profondément modifié l'approche et l'étude des polymorphismes. Il y a encore peu de temps, lorsqu'on avait choisi d'étudier un gène candidat, il fallait le séquencer, puis choisir les polymorphismes et procéder enfin aux études génétiques (études d'association, et études de liaison familiale). Avec le développement des bases de données, en particulier la base Hapmap (www.hapmap.org), on dispose aujourd'hui d'un véritable catalogue des variants génétiques communs chez I'homme. Les millions de polymorphismes identifiés sur Hapmap, permettent d'obtenir instantanément la variabilité potentielle de tout gène candidat, et ainsi de guider les analyses génétiques (études d'associations), voire d'explorer le génome entier.

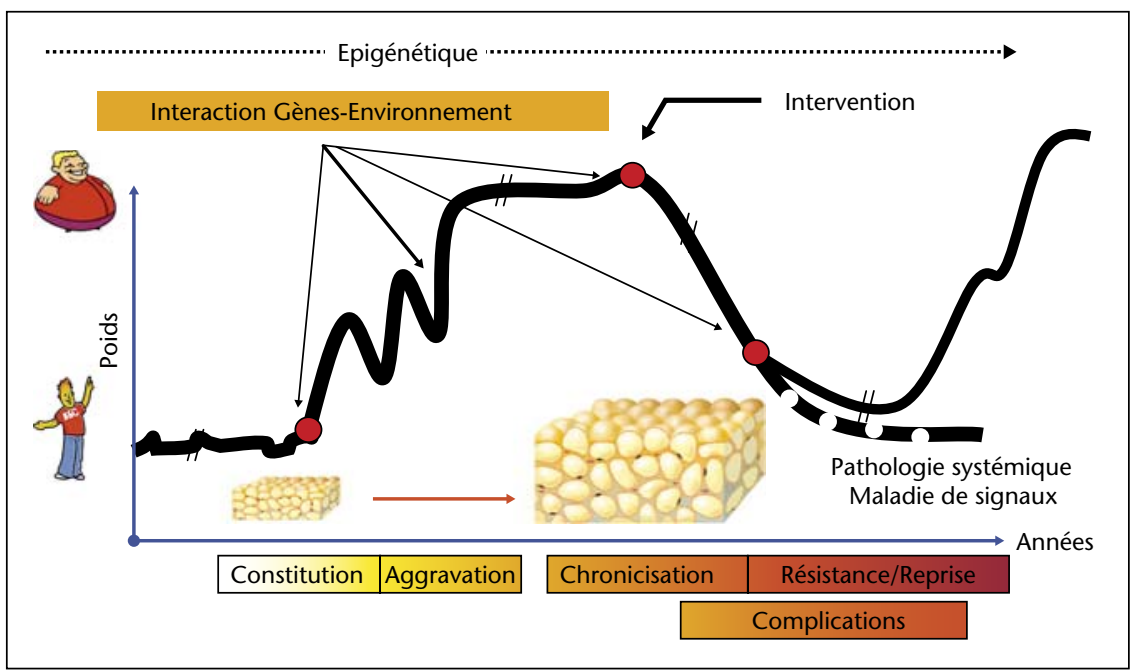

Figure 3. La prise de poids, un phénomène complexe avec différents stades de développement.
Demeure néanmoins un certain nombre de difficultés. Ces études génétiques nécessitent en effet :

- des échantillons de population de très grande taille ;

- des résultats statistiques corrigés : I'accès à des millions de SNP (single nucleotide polymorphims) pour de nombreux phénotypes reliés à l'obésité, nécessite des outils statistiques adaptés au traitement des données multiples ;

- la réplication : les polymorphismes associés à des phénotypes d'obésité ont parfois du mal à être confirmés dans des populations indépendantes;

- la validation biologique : I'analyse fonctionnelle de ces variants :

- le rôle en pathophysiologie d'un gène candidat nouveau est à définir.

\section{Les études génétiques chez I'homme: résultats}

Quelles informations fournissent ces études conduites depuis des années? L'obésité est une pathologie «polygénique» : plus de 200 régions localisées sur presque tous les chromosomes se révèlent ainsi associées à des phénotypes reliés à l'obésité tels que la masse grasse, la distribution du tissu adipeux, la variation de poids, l'occurrence d'un syndrome métabolique, l'apport énergétique, les taux de leptine et $d^{\prime}$ 'insuline circulantes, etc.

Sur la figure 4, les régions colorées représentent les régions associées à des phénotypes d'obésité dans des populations caucasiennes, américaines, européennes, les Indiens Pima, les Américains $d^{\prime}$ 'origine africaine et asiatique.

Ces résultats appellent plusieurs remarques:

- de vastes régions sont parfois concernées et contiennent des centaines de gènes; ce qui dans un premier temps les rend difficilement exploitables :

- la précision de l'analyse des régions génomiques a évolué depuis le démarrage des études, il y a une quinzaine d'années ;

- certaines régions (situées sur les chromosomes 10 et 11 par exemple) montrent des résultats convergents pour des populations indépendantes et apparaissent donc plus propices que d'autres à l'identification des «candidats »;

- enfin, difficulté inhérente à ces études: l'impossibilité de collecter au sein de ces très larges populations des phénotypes et les facteurs d'environnement avec précision.

Malgré tout, un certain nombre de gènes candidats ont pu être identifiés. Ainsi par exemple :

- GAD 2 sur le chromosome 10 est un gène responsable de la synthèse d'une enzyme, la glutamate décarboxylase, qui catalyse la production d'un neurotransmetteur (I'acide 


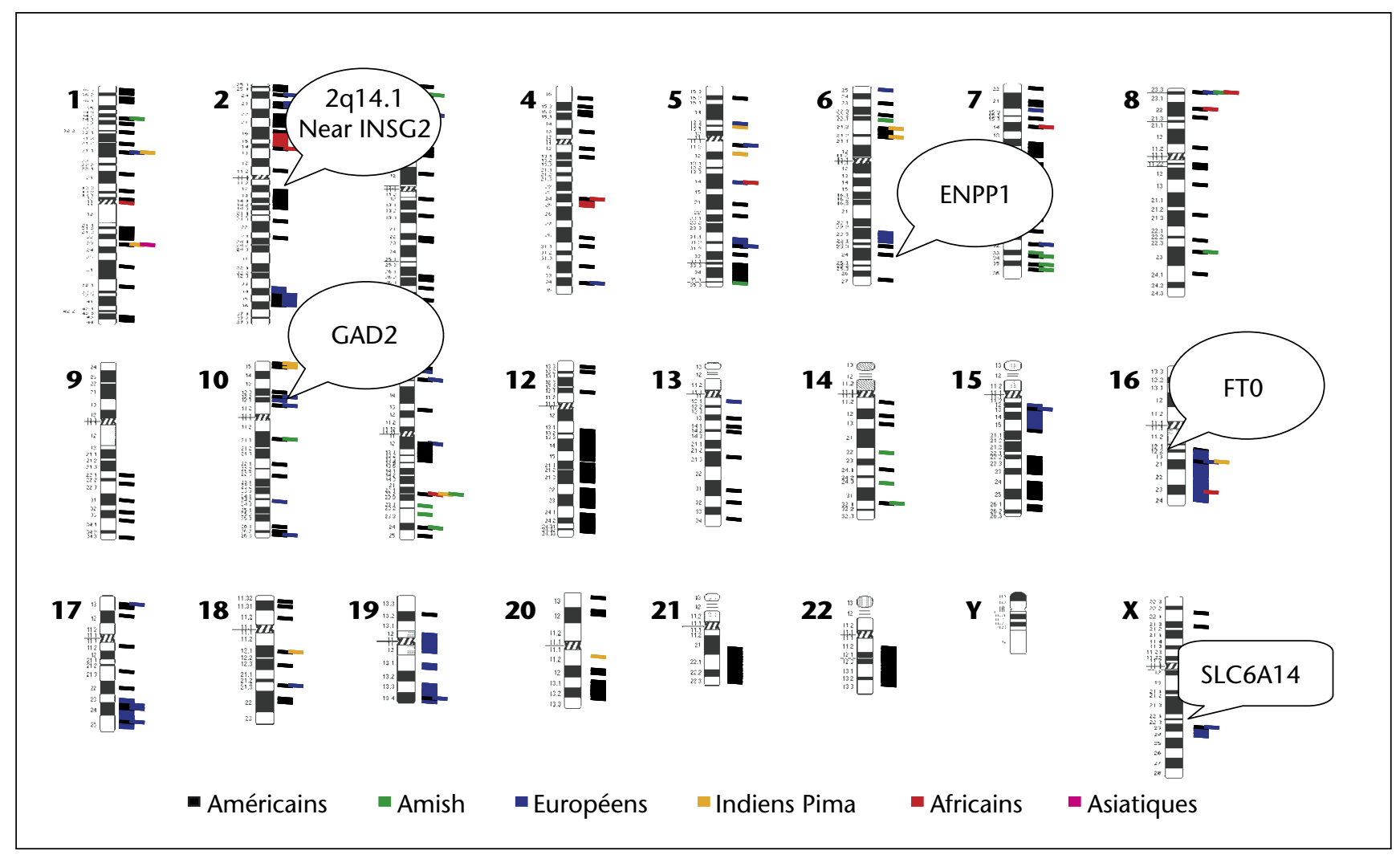

Figure 4. Obésité polygénique : loci et plus de 240 candidats. 253 QTL/244 gènes candidats (22 répliqués dans 5 études indépendantes). D'après Mutch et Clément, PLoS Genetics 2006 ; Rankinen et al. 2006.

gamma-aminobutyrique ou GABA) agissant au niveau de l'hypothalamus pour stimuler l'appétit ;

- le gène ENPP1 (ectonucleotide pyrophosphatase/phosphodiesterase 1) sur le chromosome 6 impliqué dans la signalisation de l'insuline ;

- le gène FTO (fat mass and obesity associated gene) dont le rôle est inconnu ;

- le gène SLC6A14 impliqué dans la synthèse de la sérotonine, affectant le contrôle de l'appétit.

Que dire de ces marqueurs?

Le tableau 2 montre un exemple des polymorphismes associés à des phénotypes d'obésité, certains en interaction avec l'environnement depuis 10 ans dans les populations françaises (projets soutenus par l'Assistance Publique/ Hôpitaux de Paris (AP/HP) au travers de programmes hospitaliers de recherches cliniques). Il est important de comprendre que cette liste d'allèles issus des études génétiques ne doit pas être interprétée dans un sens causal. Ces variants géniques ne sauraient à eux seuls exprimer le phénotype de l'obésité. Chacun représente au mieux un facteur de risque pour un trait particulier. Par exemple, I'allèle variant arginine (Arg) pour le récepteur $\beta 3$-adrénergique représente une augmentation de 1,7 fois du risque d'avoir une prise de poids élevée au cours de la vie dans une population particulière de sujets massivement obè- ses. L'implication de cette mutation a été très discutée et pas toujours confirmée dans les études en population dans plusieurs pays. Ces variants peuvent constituer des facteurs de ris-

Tableau 2. Facteurs de risque pour l'obésité et phénotypes associés.

\begin{tabular}{|lcc|}
\hline Gènes & Phénotypes & Odd ratios (risks) \\
\hline$\beta 3$-AR (Trp64Arg) & Prise de poids élevée & 1,7 (Clément, 1995) \\
UCP1 (-3826 A/G)* & Prise de poids élevée & 1,4 (Clément, 1996) \\
UCP1+ $\beta 3-A R$ & Prise de poids élevée & $3-4$ (Clément, 1996) \\
& Obésité massive & \\
GAD2 (haplotype) & Obésité massive & 1,30 (Boutin, PLOS, 2003) \\
PTP1b (haplotype) & Obésité & 1,49 (Coudreau, 2004) \\
& Dyslipidémie & \\
SREBP (haplotype) & Obésité massive & 1,53 (Eberlé, 2004) \\
& Diabète & \\
SLC6A14 (haplotype) & Dyslipidémies & $1,27-1,35$ (Boutin, 2004) \\
ENPP1 (haplotype) & Obésité & 1,37 (Meyre et al., 2005) \\
PPARg (Pro12Ala) & Diabète & $1,5-1,6^{*}$ (méta-analyse) \\
FTO & Diabète & $1.22-1.67^{*}(3$ études) \\
& Obésité & $(38$ participants) \\
\hline
\end{tabular}




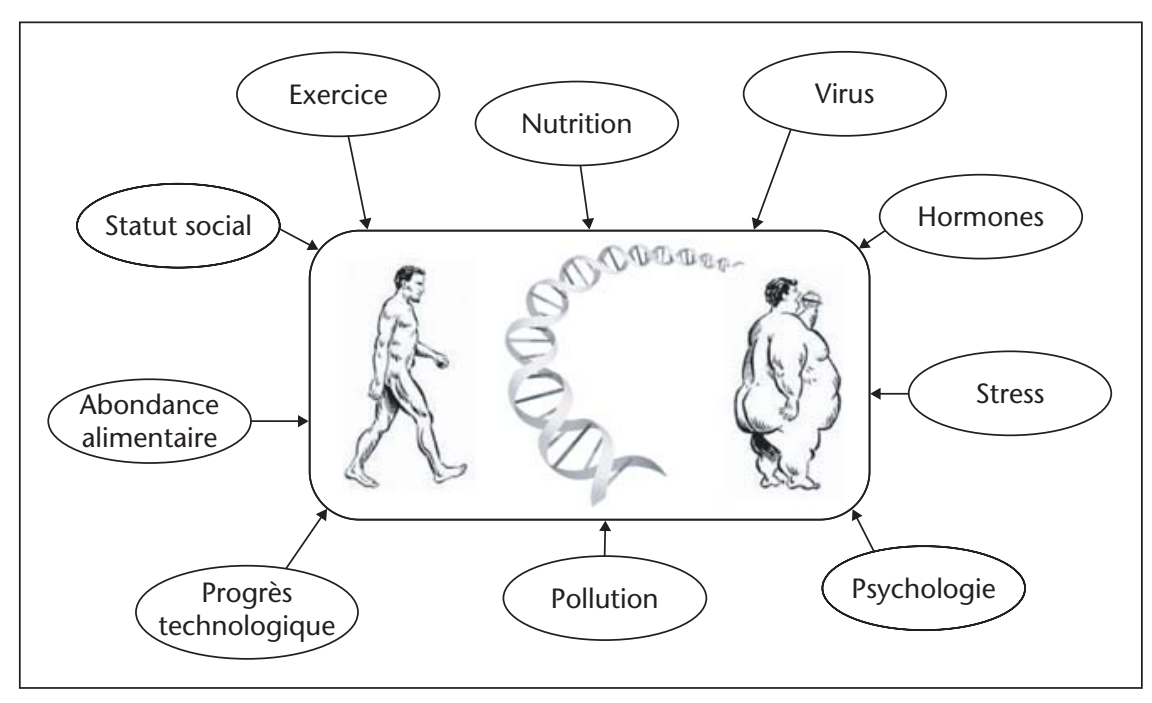

Figure 5. Interactions complexes et facteurs d'environnement. D'après Mutch D et Clement K, Plos Genetics 2006.

que relativement modestes qu'il convient de situer dans l'ensemble des facteurs de risque environnementaux; I'abondance alimentaire, la consommation de lipides, la sédentarité, le stress, le statut social, des facteurs hormonaux et bien d'autres (figure 5). Les effets de tels ou tels polymorphismes peuvent être bien différents en fonction de conditions environnementales des sujets.

\section{Les études d'interaction gènes-environnement}

Demeure donc la question des interactions complexes gènes-environnement, qui soumettent à rude épreuve nos capacités d'analyse : la multiplicité des facteurs environnementaux et génétiques à traiter, la puissance des études de population, la précision des phénotypes recueillis et également la limite de nos outils informatiques pour traiter cette complexité. Les recherches doivent se développer dans ce domaine. La précision sur les données phénotypiques se fait parfois au détriment des « grands nombres ». Ainsi dans l'étude de suralimentation menée par Claude Bouchard, chez des jumeaux, des polymorphismes géniques pourraient jouer un rôle dans la variabilité des réponses à la prise de poids. Mais il est bien difficile de conclure sur des effectifs si petits.

Conduite sur une autre échelle, l'étude européenne Nugenob (www.nugenob.com) a porté sur 750 sujets invités à consommer pendant 10 semaines un régime hypocalorique (une diminution des apports de $600 \mathrm{kcal}$ par rapport aux dépenses énergétiques de repos) dans lequel les apports lipidiques ont été modifiés. Les sujets ont été divisés en deux groupes, un groupe avec apports lipidiques "élevés »
(40-45 \%) et un avec les apports lipidiques « bas » (20-25\%). Les sujets ont été caractérisés pour des phénotypes reliés à la composition corporelle, la dépense énergétique, I'oxydation des lipides, des facteurs hormonaux et métaboliques. Une banque d'ADN a été réalisée ainsi que des prélèvements de tissu adipeux souscutané. L'objectif clairement annoncé était $d^{\prime}$ analyser I'interaction gènes-nutriments.

Après 10 semaines d'intervention, on constate dans les deux groupes une perte de poids similaire en moyenne. Ce qui confirme bien que l'apport calorique global quel que soit le bras de régime (apport lipidique élevé ou bas) est prédominant. Pour le régime avec apport lipidique bas, un plus grand nombre de sujets présentaient cependant une perte de poids supérieure à $10 \%$ et, surtout, cette étude retrouvait une grande variabilité de perte de poids entre les sujets.

Des polymorphismes de gènes candidats ont été étudiés : 42 SNP (Single Nucleotide Polymorphims ou variations d'une seule paire de base du génome), situés dans 26 gènes candidats ont été sélectionnés. II s'agissait de gènes codant pour des adipokines, des facteurs impliqués dans le métabolisme du tissu adipeux, la thermogénèse, le métabolisme lipidique et glucidique, des gènes hypothalamiques, et puis d'autres gènes candidats issus des études génome entier. II s'agissait d'évaluer, chez les porteurs de variants génétiques et chez les non-porteurs, la contribution du polymorphisme génétique dans la variabilité de la perte de poids dans l'un ou l'autre des bras de régime (effet SNP, effet régime). Ces analyses ont été contrôlées par le centre impliqué dans le recrutement, l'âge, le sexe, le poids de départ (modèle de régression) et par le nombre d'analyses (méthodes de permutation). Cinq gènes candidats parmi 26 ont montré des indications d'interaction gène-effet du régime souvent à la limite de la significativité statistique (figure 6). Les différences étaient globalement très faibles. Pour le gène hypothalamique GAD2 (sur le chromosome 10), les hétérozygotes et homozygotes porteurs du variant ne présentent qu'un excès de perte de poids de $1,8 \mathrm{~kg}$. On peut ici se poser la question de la signification de ces différences tellement l'écart est faible. Les modulations sont relativement modestes mais peut-être permettent-elles de désigner de réelles interactions gènesenvironnement pouvant jouer un rôle à long terme.

\section{Cas particulier du gène PPAR $\gamma$ ?}

Dans beaucoup d'études gènes-environnement, demeure le problème de la confirmation des résultats dans les études indépendants. Parmi les polymorphismes de gènes candidats, les plus confirmés figure la mutation Proline 12 Alanine (Pro12Ala) du gène codant pour PPAR $\gamma$.

Cette mutation Pro12Ala a été identifiée à la fin des années 90 comme ayant des conséquences fonctionnelles. En particulier sa présence dans le gène PPARy entraîne une diminution de $50 \%$ de l'activité transcriptionnelle. L'allèle Pro12 est associé au risque accru de diabète de type 2 (le niveau facteur de risque de 1,2 est même nature que ceux des autres candidats évoqués précédemment). L'allèle Ala serait quant à lui protecteur.

En étude de population on ne peut que constater encore une fois la complexité à laquelle sont confrontés les chercheurs.

Dans une population de conscrits danois (1 621 sujets; 752 obèses, 869 non obèses suivis 25 ans, Ek Diabetologia 1999). On constate chez les sujets obèses que l'allele Ala, est associé à une augmentation annuelle de I'indice de masse corporelle (IMC) supérieure à celle des sujets porteurs de l'allèle Pro. Les sujets non obèses au contraire seraient plutôt protégés contre cette prise de poids (sujets obèses Ala12Ala : $+0,27 \mathrm{~kg} / \mathrm{m}^{2} /$ an et Pro12 + $0,17 \mathrm{~kg} / \mathrm{m}^{2} /$ an, sujets non obèses Ala12Ala : $+0,11 \mathrm{~kg} / \mathrm{m}^{2} / \mathrm{an}$. Pro12 + 0,17 kg/m²/an).

Dans une population finlandaise (119 nondiabétiques (IMC $27 \mathrm{~kg} / \mathrm{m}^{2}$ ), suivi 10 ans, Lindi Diabetologia 2001). L'allèle Ala, est associé à une augmentation annuelle de I'IMC supérieure à celle des sujets porteurs hétérozygotes (I'allèle Ala12Ala : + 11,2\% poids, Pro12Ala + $5 \%$, Pro12Pro 1,8 \% d'IMC).

L'allèle Ala se révèle également associé à une reprise plus importante du poids après un régime (Niklas, Diabetes, 2001). 


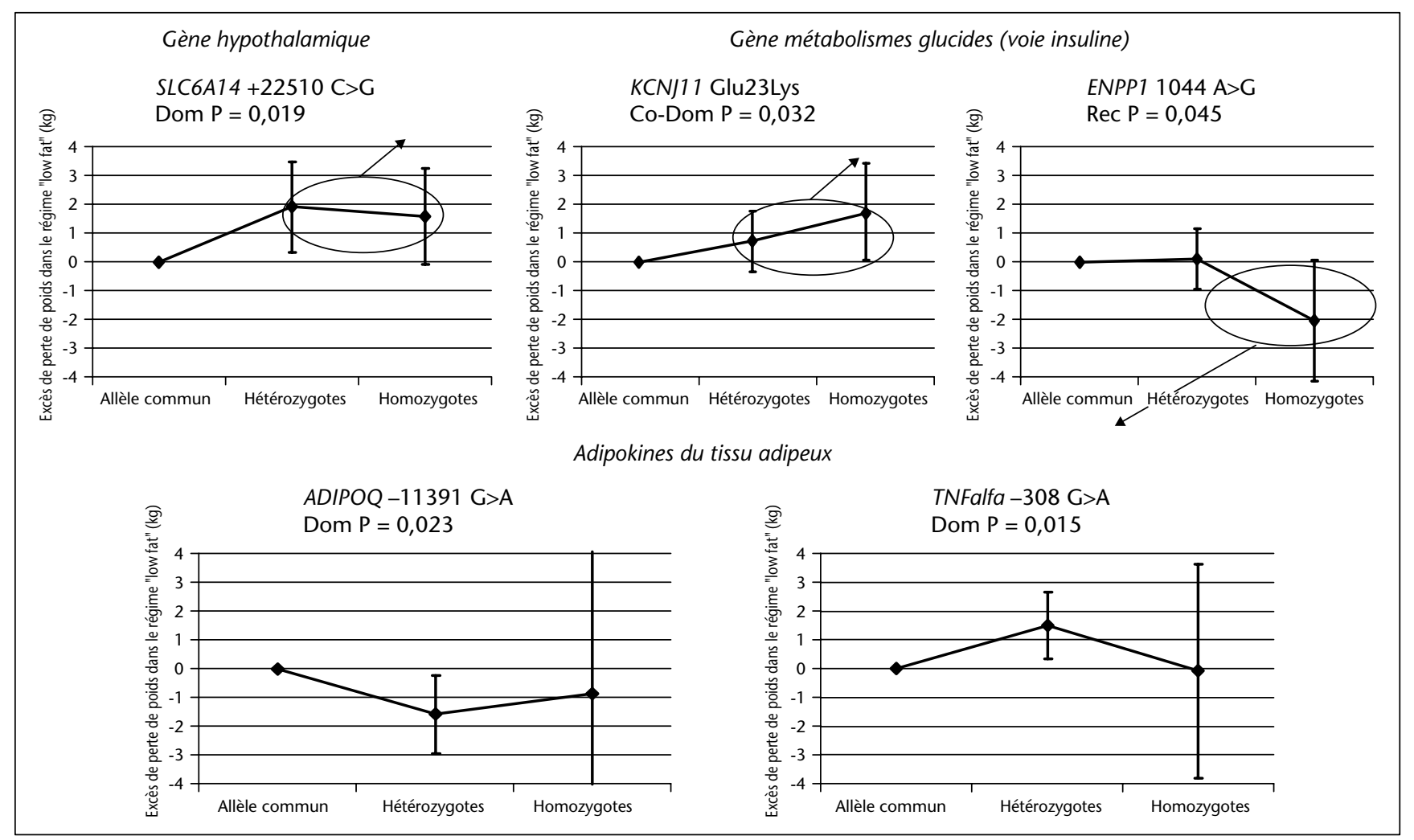

Figure 6. Excès perte de poids groupe « low-fat » chez les porteurs des variants vs. les non-porteurs des variants de gènes candidats.

Ainsi, au regard de ces études et de bien d'autres l'allèle Ala apparaît comme tantôt protecteur tantôt défavorable pour la prise de poids. En 2001, une équipe de Cambridge a publié chez 592 sujets hommes et femmes un bel exemple d'étude d'interaction gènealimentation avec ce polymorphisme candidat. Les résultats montrent que les porteurs Ala seraient plutôt protégés d'un excès d'IMC mais seulement lorsqu'ils consomment un ratio acides gras insaturés/acides gras saturés élevé. Alors que le même allèle apparaît défavorable chez les sujets ayant une consommation élevée d'acide gras saturés.

En résumé ${ }^{1}$, les interactions du Pro12Ala avec I'environnement illustrent bien la multiplicité des facteurs impliqués, comme le rôle des

${ }^{1}$ L'ensemble des informations et références citées dans ce texte peut être retrouvé dans le livre édité par l'auteur Obesity genomics and post genomics, Karine Clément et Thorkild IA Sorensen (Eds). Informa Health care. consommations en lipides (le rapport insaturés/saturés), l'interaction avec d'autres allèles du gène PPAR $\gamma$, le niveau d'activité physique, l'âge et sûrement bien d'autres. L'enjeu est de révéler les candidats et surtout les interactions pertinentes pour la santé des individus! 